\title{
Parameter analysis and design framework for magnetic adhesion wall climbing wheeled robot
}

\section{Salman Hussain*, Tariq Sattar and Ener Salinas}

Faculty of Engineering,

Science and the Built Environment,

London South Bank University,

London, UK

Email: hussas20@1sbu.ac.uk

Email: sattartp@1sbu.ac.uk

Email: salinae@1sbu.ac.uk

*Corresponding author

\begin{abstract}
Some robots need to climb ferromagnetic walls for performing important inspections and evaluations of the material properties of these walls. This paper aims to establish a design framework for magnetically adhering wheeled robots having magnets attached to the base of the robot. The different design parameters influencing the magnetic adhesion include the geometry of the flux concentrator, the variation of the air gap on adhesion and climbing performance in addition to various types of materials for magnetic flux concentration. These parameters shaping adhesion behaviour are simulated numerically using magnetostatic analysis in ANSYS Finite Elements Method (FEM) software. The results are evaluated and a set of rules and procedures are created as a framework that will enable a more efficient design and construction of this type of robots.
\end{abstract}

Keywords: wall climbing robot; magnetic flux density; ferromagnetic materials; magnetic concentrators; permanent magnets; neodymium; magnetic adhesion; robot design; NDT; non-destructive tests; static and dynamic mechanical analysis; FEM; finite elements method; ANSYS software.

Reference to this paper should be made as follows: Hussain, S., Sattar, T. and Salinas, E. (2012) 'Parameter analysis and design framework for magnetic adhesion wall climbing wheeled robot', Int. J. Intelligent Systems Technologies and Applications, Vol. 11, Nos. 1/2, pp.102-116.

Biographical notes: Salman Hussain is working at London South Bank University (LSBU) in the Group of Mechatronics, Robotics and NDT. He graduated from the Department of Mechanical Engineering at the University of Engineering and Technology, Taxila, Pakistan. He obtained a grant from this university to pursue graduate studies in UK. He made his MSc degree with a thesis in control for swimming robots. His is developing new design techniques for wall climbing robots. In particular he is studying some generalisations of magnetic adhesion techniques. He is also investigating ways to improve the payload capacity of vortex-based robots.

Tariq Sattar leads the Mechatronics, Robotics and Non-Destructive Testing Research Group in the Faculty of Engineering, Science and the Built Environment, London South Bank University. He is the Director of the Centre 
for Automated and Robotic NDT which has been at the forefront in developing wall climbing and swimming robots that provide access to test sites on large vertical structures to perform inspection.

Ener Salinas has various research backgrounds, namely theoretical physics, astrophysics, electrical and electronic engineering. Born and graduated in Peru, he obtained his $\mathrm{PhD}$ from Chalmers University in Sweden. He has been in the UK since 2002, first in the group of Physical Electronics and Materials at LSBU, then, since 2008, he is a Senior Visiting Research Fellow at LSBU in the group of Mechatronics, Robotics and NDT and is also a regular guest researcher at UNI in Lima-Peru. His current research interests are magnetism and electromagnetic design applied to various areas of science and technology.

This paper is a revised and expanded version of a paper entitled 'Towards optimum design of magnetic adhesion wall climbing wheeled robots' presented at the '26th International Conference of CAD/CAM, Robotics \& Factories of the Future (CARs\&FOF)', Kuala Lumpur, Malaysia, 26-28 July 2011.

\section{Introduction}

Climbing and walking robots perform tasks that are too difficult, dangerous or time consuming for the human worker (Sattar, 2000; Shang et al., 2008). On the one hand, among these tasks, an important one that is related to our research interests consists in carrying some evaluation equipment to perform Non-Destructive Tests (NDT) on designated wall materials. In this case it is evident that the weight of the carried equipment as a payload will add significantly to the weight of the robot itself. On the other hand, we are also interested in the analysis and inspection of relatively large structures that are often made of ferromagnetic materials (iron or steel). It is then important to have an efficient method of adhesion for the robots to attach and displace on the surface of these ferromagnetic walls. It is very convenient if this method employs this specific type of materials as an advantage and that is precisely what bringing into play permanent magnets can achieve. Furthermore, these devices should be as strong as possible. Fortunately, since its development in 1982 by General Motors and Sumimoto Special Metals, neodymium permanent magnets (made of rare earths) have been the strongest magnets ever produced. Commercially they usually go from grade N28UH to grade N52. To give an example, a square neodymium magnet grade N52 with dimensions: $50 \mathrm{~mm}$ by side and $25 \mathrm{~mm}$ thickness attached to a thick iron wall can withstand a pulling force of about 300 Newtons. The effect of an arrangement of several magnets attached to a flux concentrator must of course be much larger.

Other methods of adhesion include Pneumatic, Electromagnetic, Gecko and Vortex (Chu et al., 2010). The latter two can be discounted for they are extremely weak and unable to carry payloads. Yet, Pneumatic and Electromagnetic can be made strong too and so able to carry heavy loads. Still, they both need additional high energy to power their adhesion mechanisms. In a scheme where every watt counts, this fact makes these machines relatively less energy-efficient systems in comparison with wall climbing robots based on permanent magnets' adhesion, whose energy for the adhesion operation (in combination with the wheels friction) comes nearly 'for free' from magnets. 
An essential factor which is more and more appreciated in climbing robots is their possibility of having autonomy (in addition to carrying a payload). In this case the two competing mechanisms will have fewer chances to gain this characteristic, for it should carry an extra source for the corresponding additional power for adhesion. The adhesion by permanent magnets spares the robot of this burden. Thus, it is not unusual to design in this way autonomous climbing robots carrying relatively large payloads. This paper focuses on studying magnetic adhesion of wall climbing robots (with or without payload) to ferromagnetic walls.

There are three different magnet deployment configurations to achieve adhesion in wheeled wall climbing robots. They achieve adhesion by using magnetic wheels (Kawaguchi et al., 1995; Slocum et al., 2004; Yukawa et al., 2005; Yukawa et al., 2006; Fischer et al., 2007; Tache et al., 2007; Jin et al., 2010), magnetic tracks (Shen et al., 2006; Rochat et al., 2009; Yuanming et al., 2010) and magnets attached to the base of the body (Shang et al., 2008).

A few researchers have attempted to study optimal adhesion of robots based on magnetic wheels (Yukawa et al., 2006; Yuanming et al., 2010) and magnetic track adhesion (Shen et al., 2006), but these have been limited to specific robots. Also, such works have focused on only one or two variables affecting the design. To our knowledge, no work has been done to address an entire set of relevant variables, especially when related to robot design with magnets attached to the body of the robot. This paper addresses the possibility of a systematic study of various parameters for magnetic adhesion of wall climbing robots. This paper presents results of this study, giving in this way a useful insight into the stability of a climbing robot and it is expected to help in deciding how to achieve the best possible design.

\section{Background}

The aim of this investigation is to lay down the foundation for developing a design framework for magnetically adhering wheeled robots.

The design cycle comprises a two step process: the first step is to find the best design from the mechanical point of view, whereas the second step consists in finding the most effective adhesion. To study and evaluate climbing robot design, a static and dynamic force analysis is carried out. This analysis serves to select the most favourable design parameters by considering the adhesion force requirement, dimensions of the robot, material properties to allow selection of materials, the robot configuration and its centre of gravity. The parameters obtained from the first step provide a gateway to the second step of the design cycle.

The parameters for adhesion depend on methods to strengthen the magnetic field using a flux concentrator, the geometry of the flux concentrator, material of the flux concentrator, effect of the variation of the air gap on adhesion and climbing performance, different magnetic arrangements and the effect of wall thickness variations. These effects are studied using simulations based on Finite Element Methods (FEM) using magnetostatic analysis in ANSYS. Some of the simulation results are validated by experimental data obtained from our previous tests. 


\section{Parameter choice design}

Static and dynamic force analysis is necessary to analyse and obtain the best design parameters. In this section, static and dynamic force analysis for the wall climbing robot is carried out.

\subsection{Static analysis}

The stability of a wall climbing robot depends mainly on turnover failure, sliding failure and rollover failure as shown in Figure 1. Static analysis helps to find the design parameters to address these stability concerns.

Figure 1 Stability factors (a) turnover failure, (b) sliding failure and (c) rollover failure (see online version for colours)

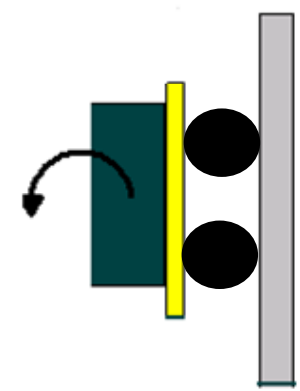

(a)

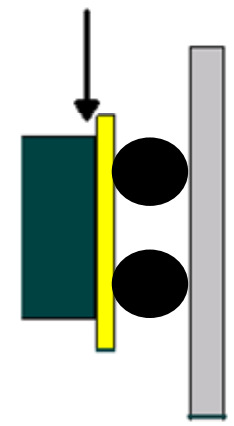

(b)

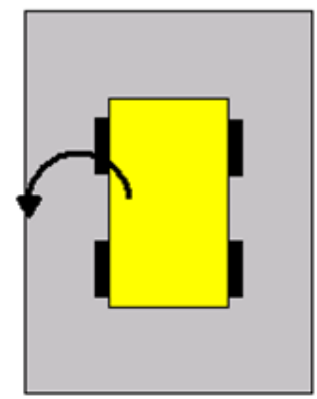

(c)

\subsubsection{Sliding avoidance}

The ideal wall climbing robot should do climbing surface transitions and climb on surfaces with different slopes. To understand the forces acting on a robot, let us consider the forces acting on a robot resting on an inclined plane as shown in Figure 2.

The slope of the inclined plane is ' $\theta$ '.

Figure 2 Free body diagram of robot moving on an inclined plane
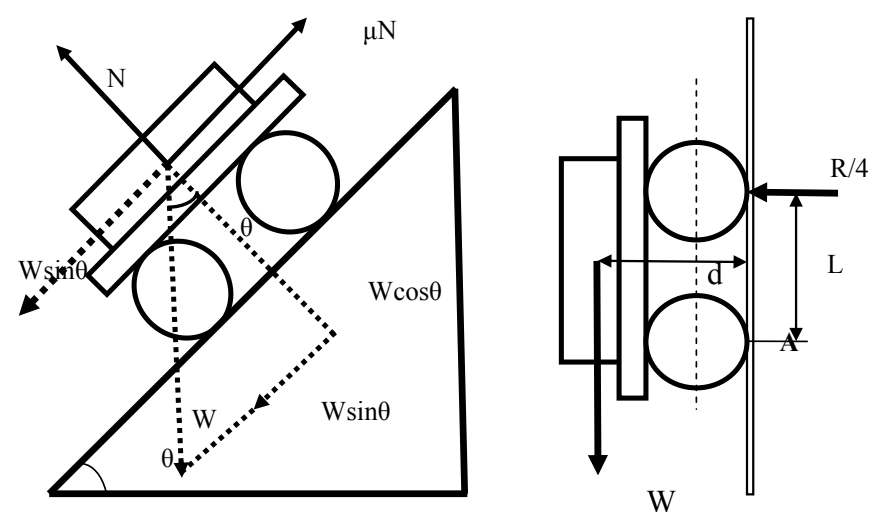


$$
\begin{aligned}
& \sum F_{y}=W \cos \theta+F_{m}-N=0 \\
& N=W \cos \theta+F_{m} \\
& \sum F_{x}=W \sin \theta-\mu N \\
& o r \\
& N=\frac{W \sin \theta}{\mu} \\
& W \cos \theta+F_{m}=\frac{W \sin \theta}{\mu} \\
& F_{m}=\frac{W \sin \theta}{\mu}-W \cos \theta
\end{aligned}
$$

where

$W=$ weight of the robot

$\theta=$ angle of inclination

$F_{m}=$ magnetic adhesion force

$\mu=$ coefficient of friction of wheels

$d=$ distance of centre of gravity from the climbing surface

$L=$ distance between front and rear wheels.

For the robot to avoid slipping

$$
F_{m}>\frac{W \sin \theta}{\mu}-W \cos \theta
$$

For the special case of a wall climbing robot moving on a vertical surface

$$
\begin{aligned}
& \theta=90 \\
& F_{m}>\frac{W}{\mu}
\end{aligned}
$$

In order to avoid sliding/slipping of the robot, the magnetic adhesion force should be greater than $W / \mu$. The stability of the robot can be increased by either increasing the coefficient of friction of wheel tyre or decreasing the robot weight.

\subsubsection{Turnover avoidance}

From Figure 2, taking moment about point A,

$$
\begin{aligned}
& \sum M=W \times d+\frac{R}{2} \times L=0 \\
& R=-\frac{2 W \times d}{L} \\
& F_{m}=R \\
& F_{m}=-\frac{W \times d}{2 L}
\end{aligned}
$$


To avoid turnover, the adhesion force should satisfy equation (2).

$$
F_{m}>\frac{W \times d}{2 L}
$$

For a given adhesion force, equation (2) can be satisfied by minimising the ratio $d / L$. This means that the centre of gravity should be as close to the surface as possible and the distance between the wheels should be large. Equation (2) shows that in order to avoid turnover, the robot centre of gravity should be kept as low as possible.

The stability criteria to avoid sliding and turnover:

$$
F_{m}>\max \left\{\frac{W}{\mu}, \frac{W \times d}{2 L}\right\}
$$

\subsubsection{Rollover avoidance}

For simplicity, we assume that the shear force on the robot always acts perpendicular to the wheel. Consider Figure 3a, the rollover moment $M_{r}$ will then be

$$
\begin{aligned}
& M_{r}=(W \times k)-2\left(F_{s} \times L_{1}\right)=0 \\
& (W \times k)=2\left(F_{s} \times L_{1}\right) \\
& 2 F_{s}=\frac{(W \times k)}{L_{1}}
\end{aligned}
$$

where

$k=$ distance between centre of gravity and point $A$

$F_{s}=$ shear force on each wheel

$L_{1}=$ moment arm, distance between wheel 4 and 1 .

Figure 3 Rollover forces when a robot is at different orientations on the wall (see online version for colours)

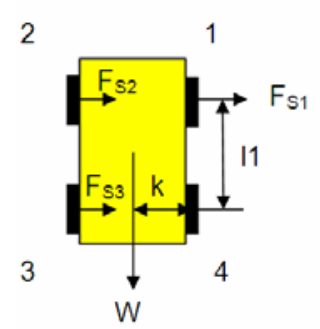

(a)

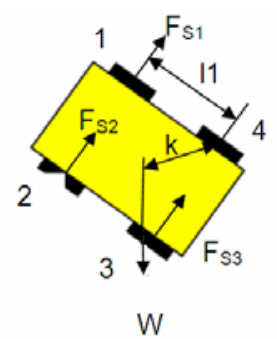

(b)

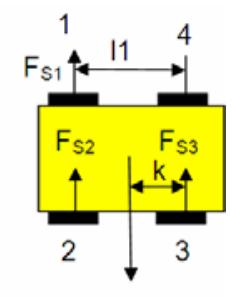

W

(c)

In order to avoid rollover,

$$
F_{s} \geq \frac{(W \times k)}{2 L_{1}}
$$

The rollover force varies with the angle of the robot on the wall (Figures $3 b$ and $3 c$ ). This is due to the variation of the moment arm with different orientations of the robot. 
The robot length (distance between the wheels) and the location of its centre of gravity are important design considerations. These parameters play an important role in determining the motion and stability of the wall climbing robot.

\subsection{Dynamic analysis}

The design for the motor torque requirement will be based on torque analysis when the robot is moving upward. This is due to the fact that the torque required for a climbing robot to move will be maximum when the robot is moving upward. Consider a robot on a wall moving upward as shown in Figure 4.

Figure 4 Moment force diagram for robot moving upward

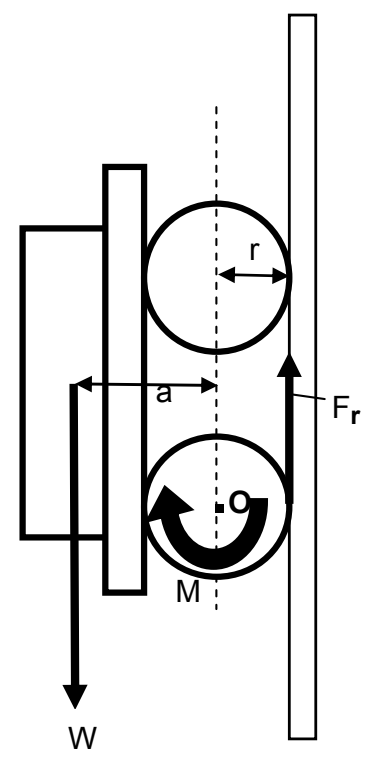

The moment about point $O$ is

$$
\begin{aligned}
& M-(W \times a)-F_{r} \times r=0 \\
& M=(W \times a)+\left(F_{r} \times r\right) \\
& F_{r}=\left(\mu \times F_{m}\right) \\
& M=(W \times a)+\left(\mu \times F_{m} \times r\right)
\end{aligned}
$$

where

$a=$ distance between centre of gravity of robot and wheel centre

$M=$ total torque required for the robot to move upward

$M_{w}=$ torque required by each wheel

$O=$ centre of the wheel

$r=$ radius of the wheel

$F_{y}=$ rolling force required 
If there are ' $w$ ' numbers of driver wheels, torque required by each wheel $M_{w}$ will be

$$
M_{w}=\frac{M}{w} .
$$

\section{Numerical simulations for magnetic adhesion}

The magnetic adhesion properties can be studied by using finite element software. We use ANSYS magnetostatic analysis. The magnets were first modelled in ANSYS design modeller. The magnet was then imported into ANSYS magnetostatic analysis. Like all the FEM procedure, the meshing of the magnets was carried out and the boundary conditions were defined and simulated. The results of the first set of simulations were verified by experimental results to validate the simulation setup and boundary conditions.

\subsection{ANSYS magnetostatic analysis}

The ANSYS magnetostatic analysis enables us to analyse different magnetic properties of the designed system. It includes flux density, field intensity, force summation, torque, energy and magnetic flux.

In Figure 5a, schematic of magnetic circuit is shown. The magnetic circuit has flux concentrator, magnets and climbing surface. The flux concentrator has limbs to direct the magnetic lines. It is desirable to design magnetic circuit with and air gap between climbing surface (usually steel) and magnets. In Figure 5b, the north poles of all three magnets are facing the flux concentrator. The magnetic lines of force travel from the north pole into the flux concentrator. To complete the magnetic circuit, these flux lines enter into the south pole after passing through the wall as shown by the arrows in Figure 5.

Figure 5 Magnetostatics analysis showing magnetic flux lines (a) modelled block, (b) magnetic flux lines inside modelled figure; the grey arrows represent the average of the main forces holding the object to the climbing surface (see online version for colours)

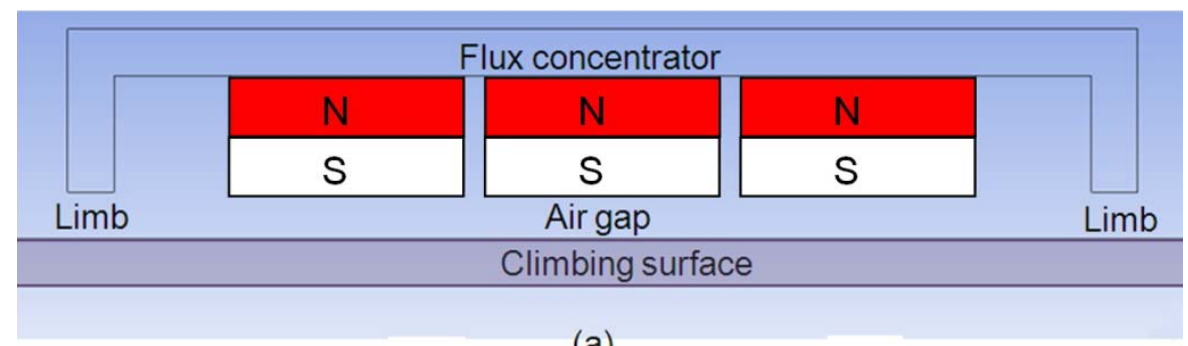

(a)

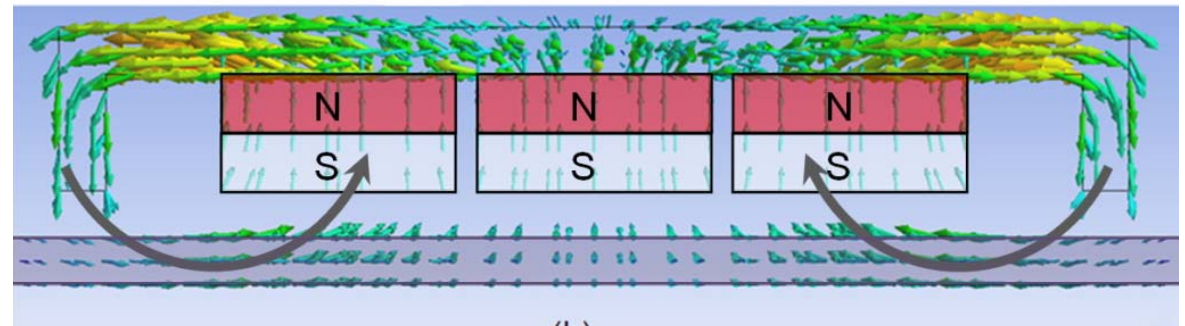

(b) 


\subsection{Validation of ANSYS magnetostatic analysis}

Two blocks of permanent magnets, one with an array of $3 \times 3$ magnets and other with array of $3 \times 2$ magnets were simulated as shown in Figure 6 . The arrays were attached to a mild steel plate. This steel plate served to concentrate the magnetic flux lines and thus increases the magnetic adhesion force. N42 magnets were used each having dimensions $50 \times 50 \times 25 \mathrm{~mm}$. The mild steel plate had a thickness of $10 \mathrm{~mm}$ and dimension of $260 \times 200 \mathrm{~mm}$ and $260 \times 160 \mathrm{~mm}$ for $3 \times 3$ array and $3 \times 2$ array, respectively.

In Figure 7, experimental results from our previous research work (Shang et al., 2008) were used to validate the results of simulations. The maximum error was found to be $8 \%$ at very high adhesion forces. This is due to the experimental apparatus capacity at high loading conditions. The overall result shows a good agreement with the experimental results.

Figure 6 Blocks of magnetic array $3 \times 2$ and $3 \times 3$ with a steel plate serving as a magnetic flux concentrator (see online version for colours)

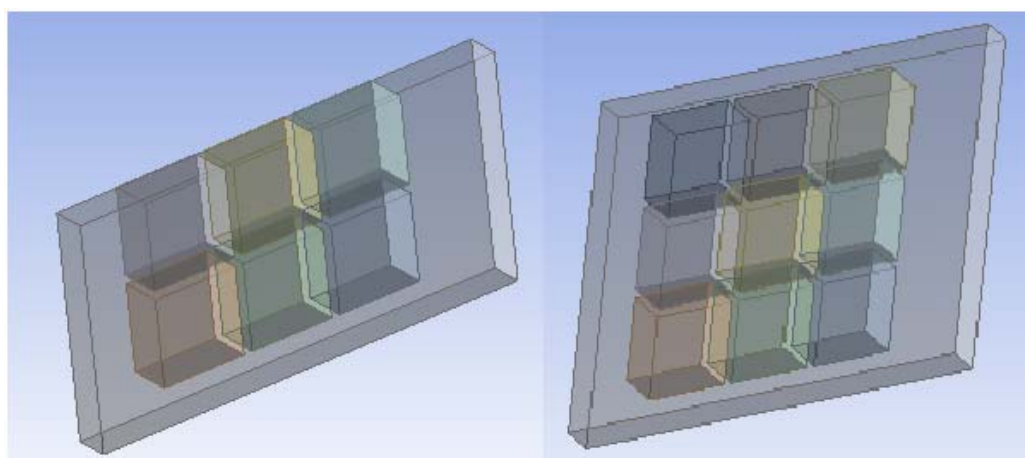

Figure 7 Magnetic force versus air gap (validation of simulation results), experimental results taken from the development phase of CROCELLS robot (see online version for colours)

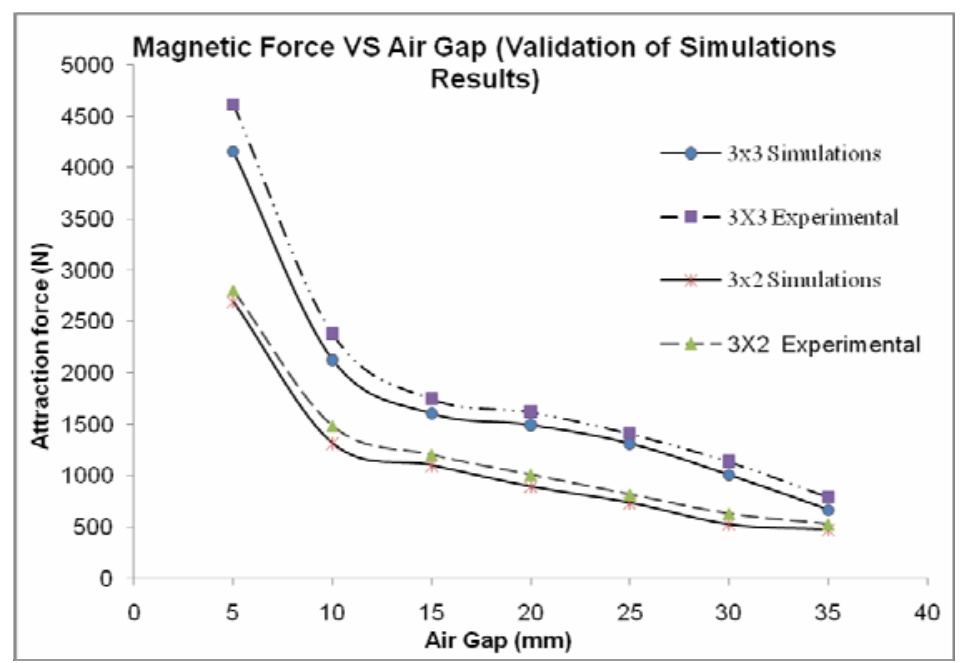

Source: Shang et al. (2008) 


\subsection{Magnetic arrangements}

Adhesion force due to different magnetic arrangements is shown in Figure 8. These magnetic arrangements include use of a flux concentrator, air gap variation from the wall surface and distance between the magnets.

The adhesion force is maximum when the magnets are $5 \mathrm{~mm}$ apart. As the distance between magnets is increased, the adhesion force starts decreasing. Thus, the closer the magnets on the robot base (flux concentrator), the higher will be the adhesion force.

Figure 8 Different magnet arrangements to achieve optimum adhesion (see online version for colours)

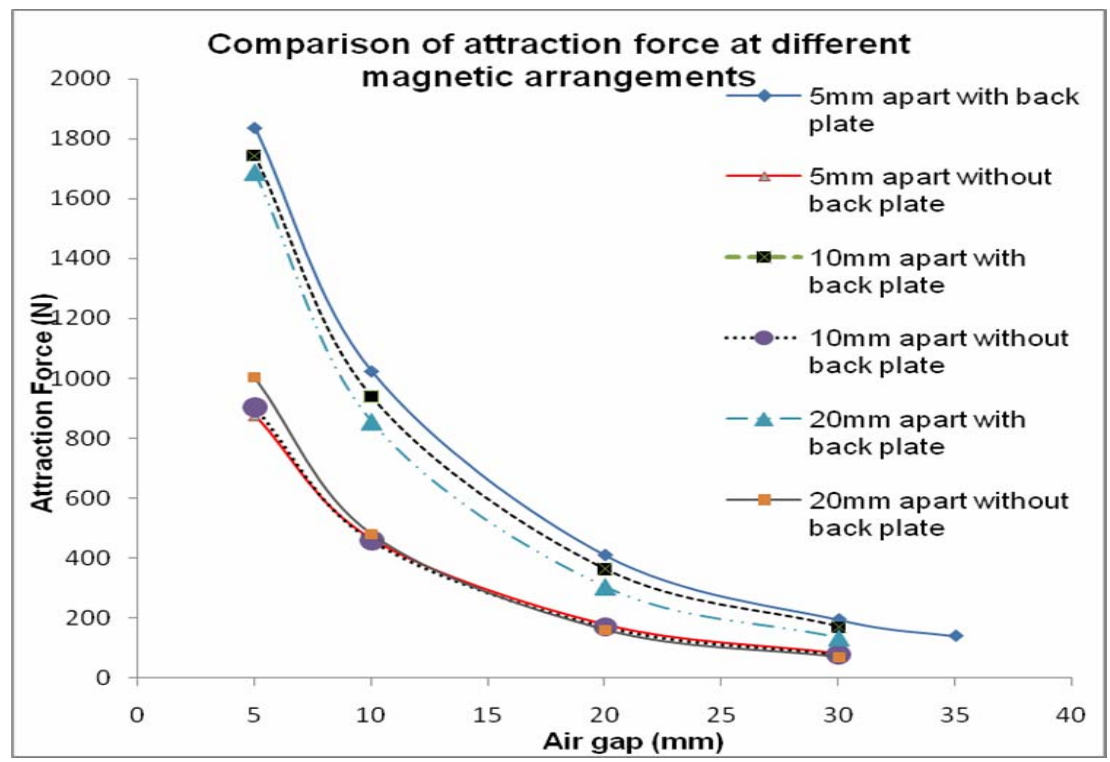

Use of the concentrating back plate (flux concentrator) shows a significant increase in magnetic adhesion as the value jumps from $1000 \mathrm{~N}$ to $1835 \mathrm{~N}$, thus almost doubling the magnetic adhesion.

The air gap refers to the distance between the face of the magnet and the wall. As this gap is increased, the magnetic adhesion decreases. This air gap is necessary to avoid obstacles in some cases and to avoid friction in all the cases. The friction at the wheel is desirable, but the friction at adhesion surfaces is not desirable.

\subsection{Effect of wall thickness}

For a specifc magnet, the wall thickness determines the ahdesion force. Simulations were carried out with a N52 magnet. The wall material used was structural steel. The adhesion force is minimum at a wall thickness of $0.1 \mathrm{~mm}$. When the thickenss of the wall is increased from $0.1 \mathrm{~mm}$ to $1 \mathrm{~mm}$, the adhesion force increases gradually. At $1 \mathrm{~mm}$, the magnetic flux is almost maximum. Any further increase in wall thickness does not have considerable effect on adhesion force as shown in Figure 9. 
Figure 9 Effect of wall thickness on magnetic adhesion (see online version for colours)

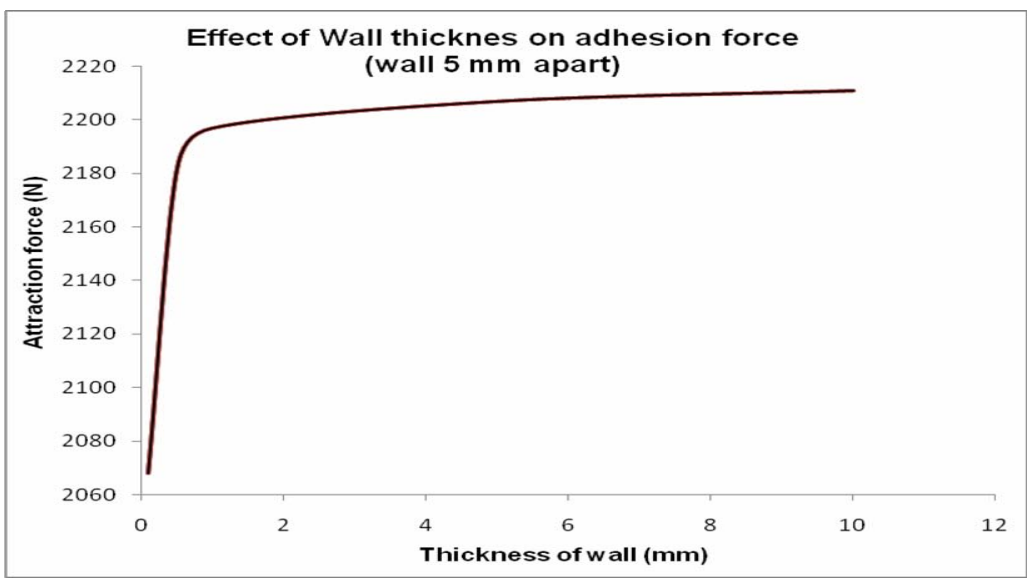

When using an air gap in the magnetic circuit, the stronger the magnet, the more adhesion force it will produce as shown in Figure 10. N28EH is the weakest material in the analysis and thus producing the lowest adhesion force. N52, being the strongest magnetic force is more desirable when circuit have air gaps.

Figure 10 Effect of air gap on different magnets having different strength

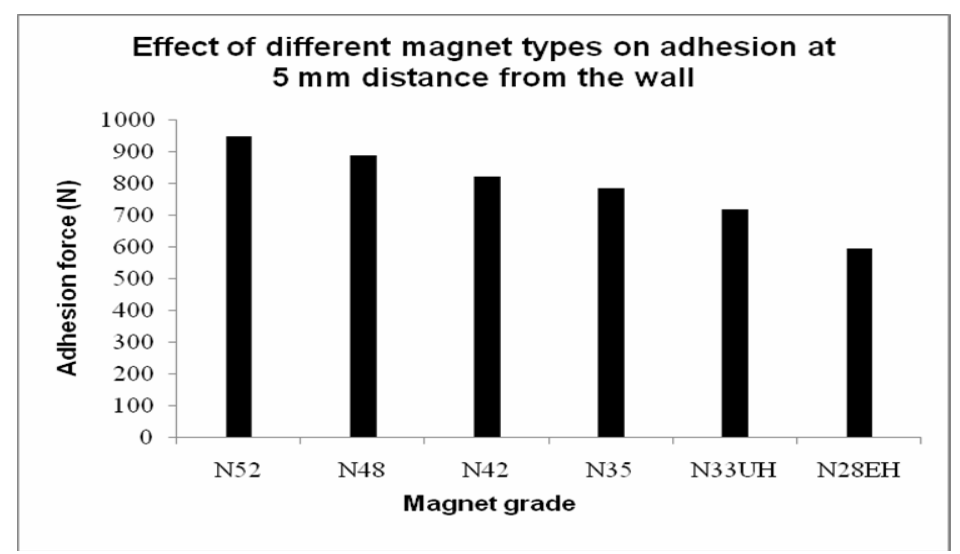

\subsection{Design of the flux concentrator}

One of the major design considerations is the design of the flux concentrator. The geometry and construction material of the concentration plate plays an important role in optimising the adhesion force. Figure 11 shows the variation of adhesion force, when different materials are used for the flux concentrator. The adhesion force is minimum when mu-metal is used and maximum when structural steel is used. The use of a flux concentrator also serves to provide strength to the chassis of the robot. So the use of structural steel is desirable though the design will perform a trade-off to reduce the weight of the climbing robot. 
Figure 11 Effect of using different material for concentration plate (flux concentrator)

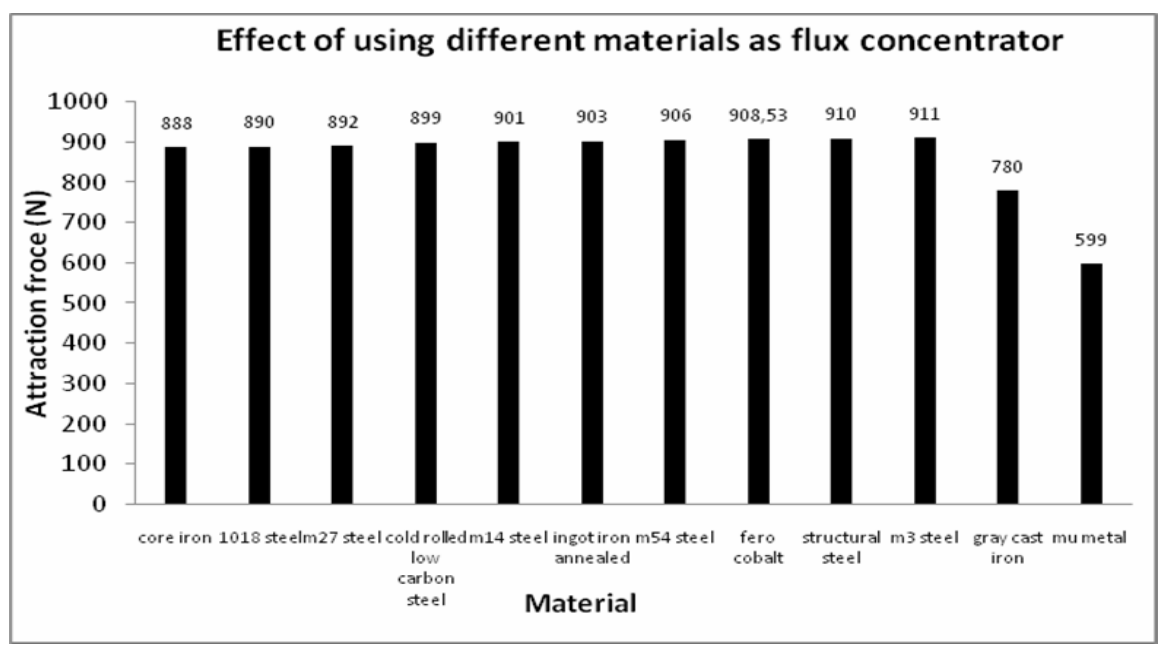

\subsection{Effect of different shape of the flux concentrator}

The flux concentrator's shape affects the magnetic flux leakage as shown in Figure 12. When the limb of the flux concentrator is skewed inward, most of the magnetic flux leaks into the south pole without passing through the wall.

Figure 12 Magnetic flux leakage due to different flux concentrator shapes (a) no limbs, (b) straight limbs, (c) limbs outwards and (d) limbs inwards (see online version for colours)

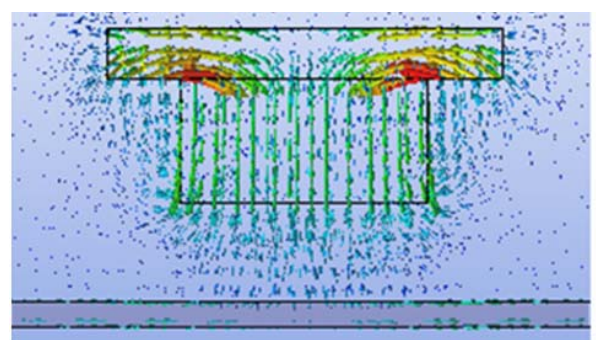

(a)

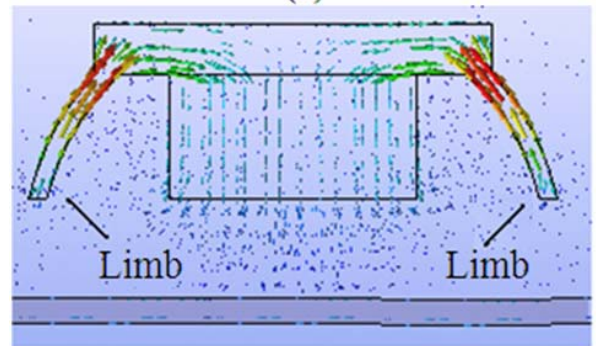

(c)

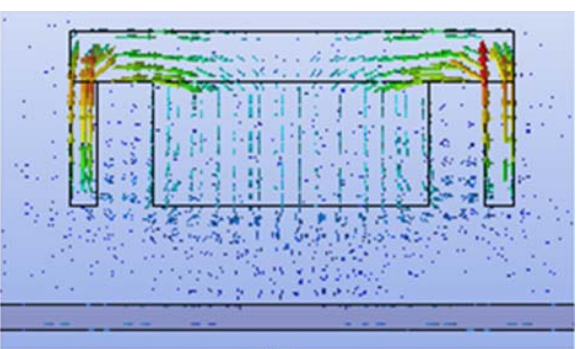

(b)

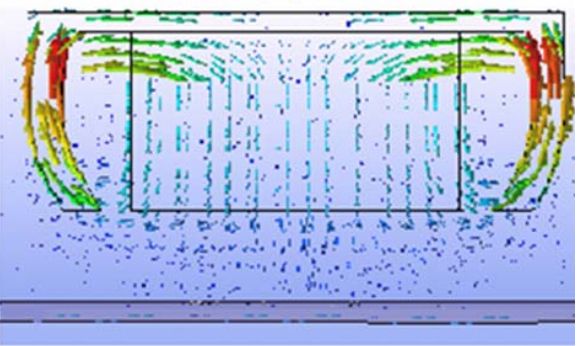

(d) 
When the limbs are straightened the magnetic flux leakage is improved but is optimum when the limbs of the flux concentrator are skewed outward. When there is no limb the magnetic flux also leaks considerably more as compared to the straight or outward limb.

Figure 13 Effect of flux concentrator shape

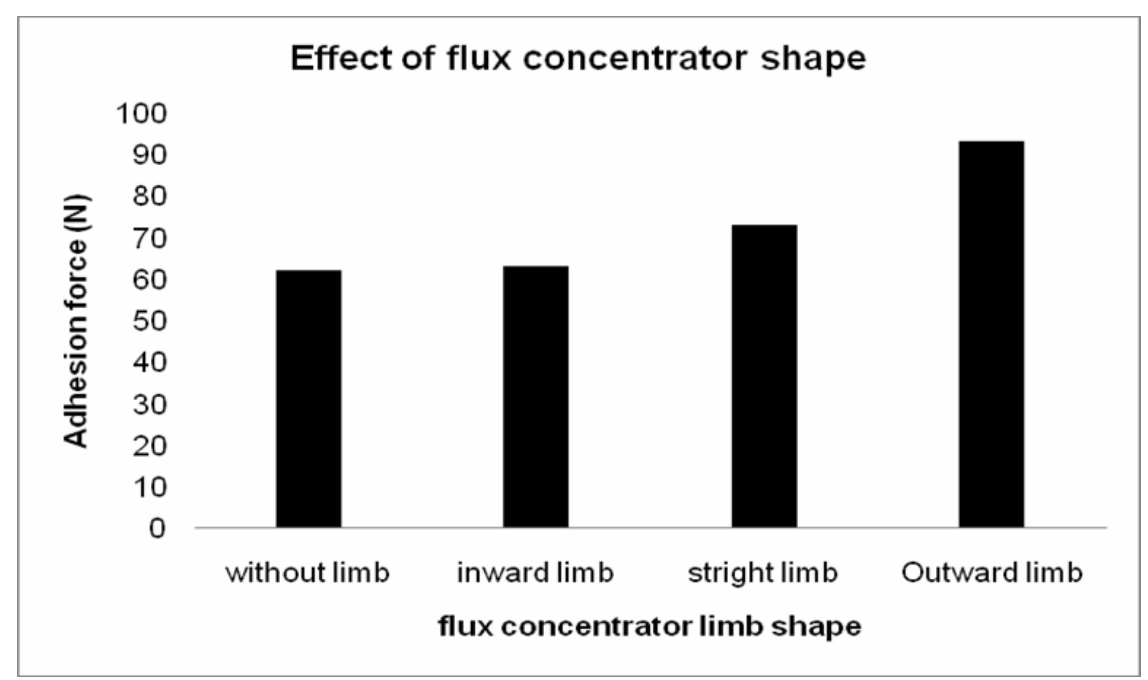

\subsection{Effect of length of the flux concentrator}

The magnetic adhesion force increases with flux concentrator size. This is due to reduction in flux leakage when the length of flux concentrator is increased. Figure 14 shows that the magnetic adhesion is proportional to the flux concentrator length.

Figure 14 Effect of length of flux concentrator on magnetic adhesion

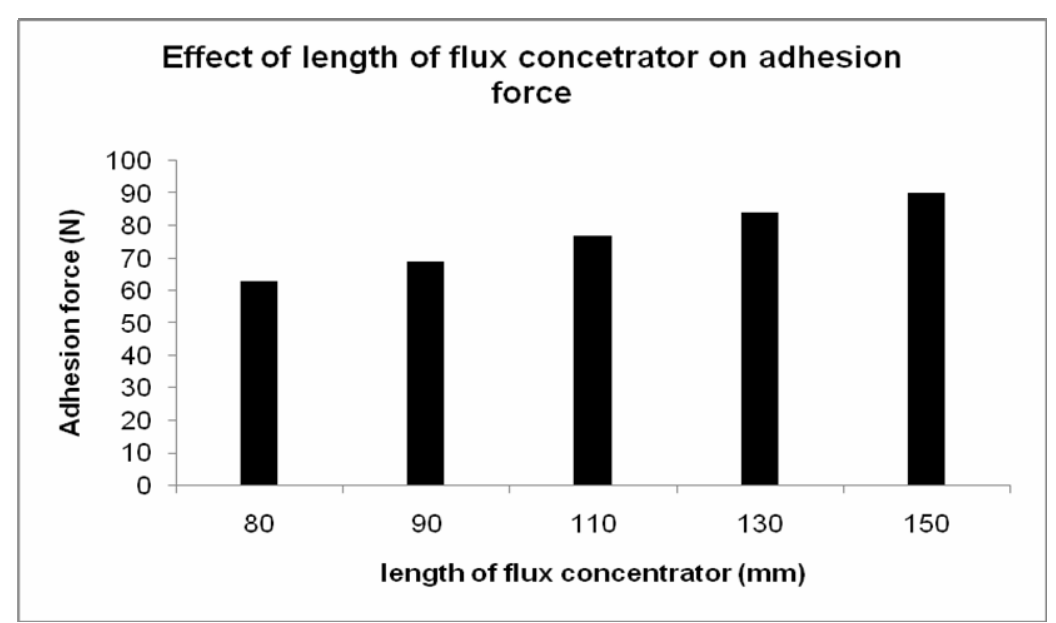




\section{Conclusions}

Different design parameters responsible for the stability of wall climbing robots were analysed. These parameters help in laying down geometric properties, material properties and configuration of the robot. FEM were used to study the optimisation of the magnetic adhesion. The study reveals the effectiveness of this approach in predicting the magnetic force for optimal design purposes. The factors affecting magnetic adhesion are the type of magnet, air gap, configuration of magnetic array, flux concentrator material, shape and length, the effect of wall thickness, parameter analysis of these factors were carried out using FEM simulations. Some of the results of the simulations were applied and validated by comparing it with the results of our robot CROCELLS (Shang et al., 2008).

These results provide a foundation to elaborate design rules to develop a wheeled robot using magnetic adhesion with magnets attached to the base of the body. An example of these rules follows:

First, a number of magnets (e.g. six) with a determined shape and weight are assumed. Free variable parameters are the grade of the magnets and geometry of the concentrators. Little should affect the position of the centre of the gravity. In addition, another free parameter is the distance between the wheels.

Second, a robot type is given according to its application. If the payload is light, the weight and the centre of gravity is mainly that of the robot itself. However, if the payload is heavy, one should include the weight of this payload and find the new centre of gravity. Now the total weight of the climbing robot plus payload is determined.

Third, the necessary adhesion force for the system robot-payload to become attached to the ferromagnetic wall is computed numerically. The main constraints are the weight and the centre of the gravity. Here, the results give the grade of the magnets and this is trade-off with the distance between wheels and the flux concentrator geometry. Along this procedure, we try to keep the friction coefficient constant.

Summarising, the input parameters are the centre of gravity, friction coefficient, distance between wheels, flux concentrator geometry and magnet grade. And the output is the necessary force to maintain the robot attached to the ferromagnetic wall. It is worth noticing that we have maintained three parameters constant (friction coefficient, number of magnets and their size). In the same way other parameters can be considered constant and the remaining adjustable. Yet, the final output parameter is always the same, i.e. the already mentioned attaching force.

Prototypes are planned to be constructed based on these results. Also a benchmark will be made, comparing these robots with the ones based on magnetic wheel adhesion, which will have an analogous algorithm to obtain the necessary adhesion force.

\section{References}

Chu, B., Jung, K., Han, C. and Hong, D. (2010) 'A survey of climbing robots: locomotion and adhesion', International Journal of Precision Engineering and Manufacturing, Vol. 11, pp.633-647.

Fischer, W., Tâche, F. and Siegwart, R. (2007) 'Magnetic wall climbing robot for thin surfaces with specific obstacles', 6th International Conference on Field and Service Robotics, pp.551-561.

Jin, Y., Chen, J. and Li, Z. (2010) 'A magnetic wheel structure for an omni-directional microrobot to limit slip effect', International Journal of Advanced Robotic Systems, Vol. 6, pp.277-283. 
Kawaguchi, Y., Yoshida, I., Kurumatani, H., Kikuta, T. and Yamada, Y. (1995) 'Internal pipe inspection robot', IEEE International Conference on Robotics and Automation, Vol. 11, pp.857-862.

Rochat, F., Schoeneich, P., Nguyen, O.T.D. and Mondada, F. (2009) 'Tripillar: miniature magnetic caterpillar climbing robot with plane transition ability', 12th International Conference on Climbing and Walking Robots and the Support Technologies for Mobile Machines (CLAWAR), Vol. 1, pp.343-350.

Sattar, T.P. (2000) 'Wall climbing crawlers for nondestructive testing', Miniature Robotics and Sensors for Non-destructive Evaluation and Testing, Vol. 4, pp.77-100.

Shang, J., Bridge, B., Sattar, T., Mondal, S. and Brenner, A. (2008) 'Development of a climbing robot for inspection of long weld lines', Industrial Robot: An International Journal, Vol. 35, pp.217-223.

Shen, W., Gu, J. and Shen, Y. (2006) 'Permanent magnetic system design for the wall-climbing robot', Applied Bionics and Biomechanics, Vol. 3, pp.151-159.

Slocum, A., Lang, J., Awtar, S., Hart, A.J. and Mukaddam, K. (2004) 'Magnetically preloaded wheels', International Conference of the European Society of Precision Engineering and Nanotechnology, pp.368-369.

Tache, F., Fischer, W., Siegwart, R., Moser, R. and Mondada, F. (2007) 'Compact magnetic wheeled robot with high mobility for inspecting complex shaped pipe structures', IEEE/RSJ International Conference on Intelligent Robots and Systems, pp.261-266.

Yuanming, Z., Dodd, T., Atallah, K. and Lyne, I. (2010) 'Design and optimization of magnetic wheel for wall and ceiling climbing robot', International Conference on Mechatronics and Automation (ICMA), pp.1393-1398.

Yukawa, T., Okano, H. and Komatsubara, S. (2005) 'Mechanisms for the movement of piping inspection robot with magnetic elements', 3rd International Conference on Computing, Communications and Control Technologies, Vol. 1, pp.289-294.

Yukawa, T., Suzuki, M., Satoh, Y. and Okano, H. (2006) 'Design of magnetic wheels in pipe inspection robot', IEEE International Conference on Systems, Man and Cybernetics, pp.235-240. 